\title{
Robust Control of Wave Energy Converters
}

\author{
Francesco Fusco $^{1}$ and John V. Ringwood ${ }^{2}$
}

\begin{abstract}
Energy-maximising controllers for wave energy devices are normally based on linear hydrodynamic device models. Such models ignore nonlinear effects which typically manifest themselves for large device motion (typical in this application) and may also include other modelling errors. In this paper, we present a methodology for reducing the sensitivity to modelling errors and nonlinear effects by the use of a hierarchical robust controller, which also allows good energy maximisation to be recovered through a passivity-based control approach.
\end{abstract}

\section{INTRODUCTION}

The use of energy-maximising control has been accepted as crucial to the development of economic wave-energy conversion (WEC) [1], allowing the effective bandwidth of WECs to be increased, generating a near resonance condition at a wide range of wave frequencies.

Despite the prevalent use of linear models in WEC evaluation, simulation and control [2], [3], there is an acknowledgement that such models are relatively simplistic in their representation of many nonlinear effects, including nonlinear Froude-Krylov forces [4], [5] and viscous drag forces [6], [7]. In particular, the concept of linearisation around an equilibrium point (the zero displacement point) where operation is in the region of this equilibrium point is often violated, since the objective is to amplify the WEC motion (via resonance) in order to maximise energy capture. The achievement of resonance also produces large device velocities (particularly in large seas), resulting in significant viscous drag forces and vortex shedding. The danger is that energy maximising controllers based on linear models may become significantly mismatched with the real WEC dynamics over significant portions of the operational space, if such controllers are sensitive to variations in the model. The result of such a mismatch will be significant reduction in energy capture, with consequences for the economic performance of the WEC device.

However, linear models are attractive due to intuitive connection with physical device quantities, their compact algebraic representation (permitting model-based control design) and their relatively low computational overhead. An ideal situation, therefore, is that we utilise a linear model for WEC control, but address the issues associated with a lack of fidelity of the model for more significant device motion. This is addressed as follows:

\footnotetext{
${ }^{1}$ Francesco Fusco is with the Smarter Cities Technology Centre, IBM Research Ireland (e-mail: francfus@ie.ibm.com)

${ }^{2}$ John Ringwood is with the Centre for Ocean Energy Research, National University of Ireland Maynooth, Maynooth, Co Kildare, Ireland (web:www.eeng.nuim.ie/coer; e-mail: john.ringwood@eeng.nuim.ie)
}

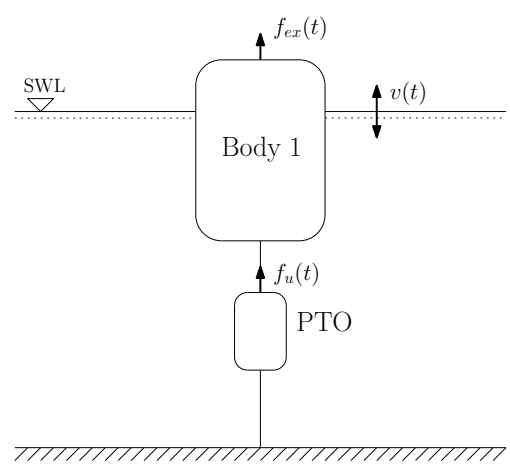

Fig. 1. One-degree of freedom floating system for wave energy conversion

- A optimal velocity reference generation controller is determined which is only weakly dependent on the WEC model, and

- A robust servo-controller is employed to address model uncertainty.

Such a concept has the advantages that the poor sensitivity and robustness properties of feedforward control (used in reference generation) are minimised, since the reference generation is only weakly dependent on the WEC model, while model uncertainties can be directly addressed using a robust feedback structure used to achieve the desired device velocity.

The reference-generation feedforward component was studied in [8]. The robust feedback controller, which deals with system non-linearities, is based on the concept of passivity and allows the achievement of robust stability as well as performance, without requiring a model of the nonlinearities. While a less conservative non-linear controller could be designed (for example by extending the linear model predictive control (MPC) designs proposed in [9][12]), an accurate model of the non-linearity, for control purposes, is not always easy to obtain and model uncertainties are still present, in practical situations. In addition, nonlinear MPC controllers generally involve iterative calculations and the inclusion of a nonlinear model accentuates the convergence issues associated with WEC MPC controllers [12].

\section{WAVE ENERgy Converter Model}

\section{A. Hydrodynamic model}

A single-body floating system oscillating in heave is considered, as shown in Fig.1. Energy is extracted from the relative motion with the sea bottom, through a generic PTO mechanism. The external forces acting on the WEC are the excitation from the waves and the control force produced by 
the PTO, namely $f_{e x}(t)$ and $f_{u}(t)$. Additional hydrodynamic and hydrostatic forces arising due to the motion of the body in the water, are the radiation force, $f_{r}(t)$, the viscous force, $f_{v}(t)$, and the buoyancy, $f_{b}(t)$ [3]. The equation of motion, in one degree of freedom, excluding mooring forces, is specified as follows:

$$
M \dot{v}(t)=f_{r}(t)+f_{v}(t)+f_{b}(t)+f_{e x}(t)+f_{u}(t),
$$

where $v(t)$ is the heaving velocity and, under the assumptions of linear potential theory [3]:

$$
\begin{aligned}
& f_{e x}=\int_{-\infty}^{t} h_{e x}(\tau) \eta(t-\tau) d \tau \\
& f_{r}(t)=-\int_{0}^{t} z_{r}(\tau) v(t-\tau) d \tau \\
& f_{b}(t)=-\rho g S_{w} \int_{0}^{t} v(\tau) d \tau \\
& f_{v}(t)=0 .
\end{aligned}
$$

In (2), the excitation (including diffraction) force is related to the incident wave, $\eta(t)$, through the excitation kernel function $h_{e x}(t)$. Equation (3) expresses the radiation force as a linear convolution of the radiation kernel, $z_{r}(t)$, with the oscillation velocity. The buoyancy, $f_{b}(t)$, models the hydrostatic equilibrium, related to the heave position through a linear coefficient that depends on the acceleration due to gravity, $g$, the water density, $\rho$ and the surface area of the body cut at the mean water level, $S_{w}$. Note the non-causality of the expression for the excitation force, where $h_{e x}(t) \neq 0$ for $t \leq 0$ [3].

However, the assumptions of linear potential theory are not necessarily valid for oscillating WECs which are subject to significant motions around the mean water level. Several studies have been carried out in order to include quadratic terms of the fluid potential and the variations in time of the wetted surface [5], [13]. Simulation results clearly show how the linear model consistently over-estimates the body motion, particularly in large waves. However, an explicit expression of the hydrodynamic forces that account for such non-linearities has not, to date, been derived.

Another unacceptable assumption, in practical situations, is the absence of viscous forces. An experimental law was proposed by Morison [14]:

$$
f_{v}(t)=\rho R C_{d}|v(t)| v(t)+\rho \pi R^{2} C_{i} \dot{v}(t),
$$

where $\rho$ is the water density, $R$ is the cylinder radius, $C_{d}$ is the drag coefficient and $C_{i}$ is the inertia coefficient. The Morison equation has been experimentally validated and methods have been proposed to evaluate the coefficients $C_{d}$, $C_{i}$ for certain specific shapes [6], [7].

In this study, we assume that the non-linearity of the system comes from the drag component of the viscous force (the first term in (6)), noting that a non-zero $C_{i}$ would only result in an additive constant to the mass, $M$, in (1)). The heaving cylinder of Fig. 1 is therefore simulated by the following non-linear model:

$$
\begin{aligned}
M \dot{v}(t)+\int_{0}^{t} z_{r}(\tau) v(t-\tau) d \tau+K_{v}|v(t)| v(t)+ \\
K_{b} \int_{0}^{t} v(\tau) d \tau=f_{e x}(t)+f_{u}(t),
\end{aligned}
$$

where $K_{v} \triangleq \rho R C_{d}, K_{b} \triangleq \rho g S_{w}$, and it is assumed that $v(t)=0$ for $t \leq 0$.

\section{B. Linear model for control design}

For convenience of control design, the force-to-velocity model of a WEC is typically expressed using a linear model in the frequency domain [3], [8]:

$$
\frac{V(\omega)}{F_{e x}(\omega)+F_{u}(\omega)}=\frac{1}{Z_{i}(\omega)} \triangleq P_{n}(\omega),
$$

where $Z_{i}(\omega)$ is termed the intrinsic impedance of the system and $P_{n}(\omega)$ will be referred to as the nominal model. In (8), $V(\omega), F_{e x}(\omega)$ and $F_{u}(\omega)$ represent the Fourier transform of the velocity, $v(t)$, excitation force, $f_{e x}(t)$ and control force $f_{u}(t)$, respectively. Note that, in the following, unless stated otherwise, the Fourier transform of time-domain signals or functions will be denoted by the corresponding capital letter, namely $X(\omega) \triangleq \mathcal{F}\{x(t)\}$.

The intrinsic impedance, $Z_{i}(\omega)$, of the model in (8) is specified as (refer to [3], [8] for the full derivation):

$$
Z_{i}(\omega)=B(\omega)+K_{v}^{(0)}+\jmath \omega\left[M+M_{a}(\omega)+M_{\infty}-\frac{K_{b}}{\omega^{2}}\right],
$$

where $B(\omega)$ is the radiation resistance (real and even [3], [15]), $M_{a}(\omega)+M_{\infty}$ is the added mass (the constant term at infinite frequency, $M_{\infty}$, is separated to yield a well-defined Fourier transform of $z_{r}(t)$ [3]) and $K_{v}^{(0)}$ is a linear damping coefficient approximating the nonlinear viscous damping in (7). By way of comparison with the nonlinear model shown in (7), the time-domain version of the linearised model can be expressed as:

$$
\begin{aligned}
& M \dot{v}(t)+\int_{0}^{t} z_{r}(\tau) v(t-\tau) d \tau+K_{v}^{(0)} v(t)+ \\
& K_{b} \int_{0}^{t} v(\tau) d \tau=f_{e x}(t)+f_{u}(t),
\end{aligned}
$$

The model in (8) allows the derivation of conditions for optimal energy absorption and the intuitive design of the feedback controller in the frequency domain [3], [8]. This study shows how the simple design of the controller based on the linear model, can be made robust to uncertainties stemming from unmodelled non-linearities.

The WEC system considered in this study consists of a heaving cylinder with radius $R=7 \mathrm{~m}$, height $H=20 \mathrm{~m}$, draught $h=16 \mathrm{~m}$, mass $M=2.54 \times 10^{6} \mathrm{Kg}$. The radiation and excitation transfer functions, $H_{r}(\omega)$ and $H_{e x}(\omega)$, are identified numerically through the hydrodynamic software Wamit [16]. The drag coefficient is set to $C_{d}=1$, based on the numerical study in [6]. The linear viscous coefficient is set to $K_{v}^{(0)}=3.17 \times 10^{5} \mathrm{Kg} / \mathrm{s}$, such that the linear model 


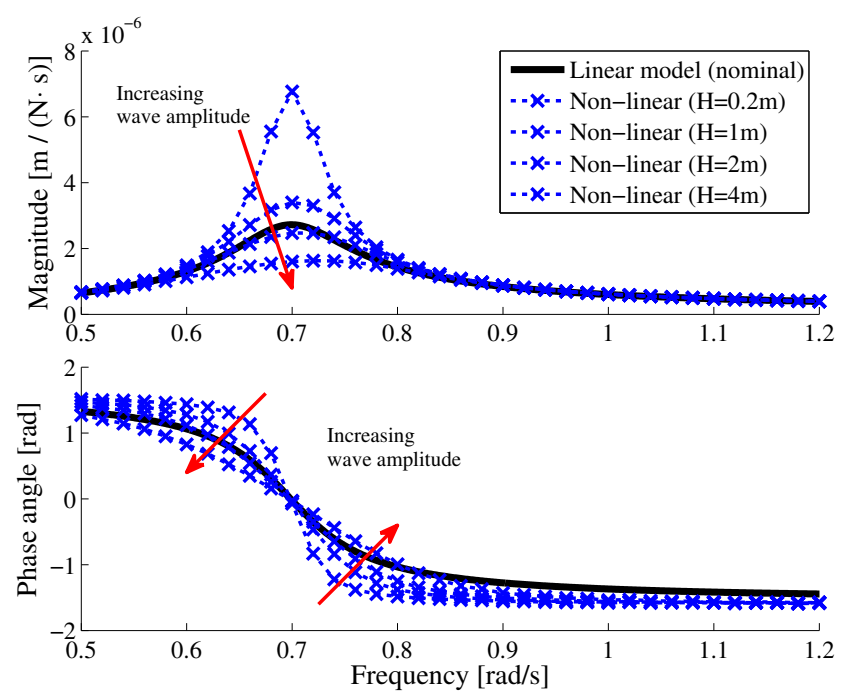

Fig. 2. Comparison between frequency response of linear system and non-linear system at waves of different amplitudes.

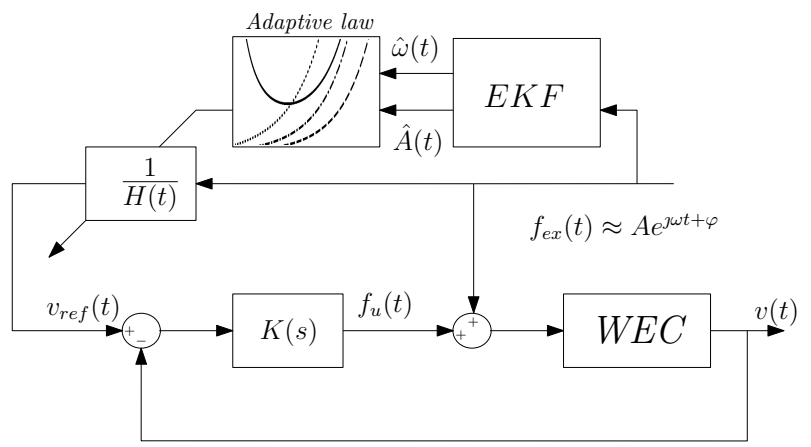

Fig. 3. Architecture of the hierarchical controller. The reference velocity is calculated by applying the adaptive the gain $1 / H(t)$ to the excitation force, based on (13).

is accurate when excited by waves of about 2 meters in amplitude.

Figure 2 compares the magnitude and phase response of the non-linear model in (7), calculated with waves of different height and frequency, against the linear model in (8). The 'frequency response' of the non-linear system is evaluated in the sense of a describing function [17], as the complex amplitude-dependent ratio of the output fundamental to the input sinusoid, evaluated based on time-domain simulations.

\section{CONTROL SYSTEM DESIGN}

The control system architecture, shown in Fig. 3, was firstly introduced in [8], in the context of WECs. Based on the wave excitation force, a high-level controller calculates a reference velocity such that the energy absorption is maximised and the motion is within desired constraints. The reference velocity is then imposed on the WEC through a low-level feedback controller that acts on the PTO force.

Section III-A gives an overview of the high-level controller (the reader is referred to [8] for the full details). The design of the low-level controller, which is the main focus of this study, is then dealt with in section III-B.

\section{A. High-level control}

It is a well known result that maximum wave power absorption from the WEC is achieved when [3]:

$$
V(\omega)=\frac{1}{2 B(\omega)+2 K_{v}^{(0)}} F_{e x}(\omega),
$$

which gives the optimal amplitude and phase of the oscillating velocity in terms of the excitation force and the hydrodynamic properties of the system. However, non-causality [3], [15], [18] and excessive requirements in terms of motion/forces [3], [8], [9] make condition (11) impractical for many situations.

Based on the assumption that the wave excitation force is a narrow-banded harmonic process with a time-varying frequency, $\omega(t)$, and amplitude, $F_{e x}(t)$, the reference velocity is calculated as a linear proportion of the wave excitation force:

$$
v_{r e f}(t)=\frac{1}{H(t)} f_{e x}(t),
$$

where $H(t)$ is adapted, on-line, according to the rule [8]:

$$
\frac{1}{H(t)}=\left\{\begin{array}{ll}
\frac{1}{2 B(\hat{\omega})+2 K_{v}^{(0)}} & \text { if } \frac{\hat{\omega} X_{\text {lim }}}{\hat{F}_{\text {ex }}}>\frac{1}{2 B(\omega)+2 K_{v}^{(0)}} \\
\frac{\hat{\omega} X_{\text {lim }}}{\hat{F}_{\text {ex }}} & \text { otherwise }
\end{array},\right.
$$

such that optimal power absorption is achieved, under the motion constraint $|x(t)| \leq X_{\text {lim }}$ [8]. The estimation of $\hat{\omega}(t)$ and $\hat{F}_{e x}(t)$ are obtained by use of the extended Kalman filter (EKF) applied to the true excitation force signal, $f_{e x}(t)$, assumed known [8], [18] (in practice it would have to be estimated from motion and/or wave-elevation sensors).

The reference velocity, calculated from (12) and (13), is weakly dependent on the model of the system, which will result in close-to-optimal performance (assuming that the estimates of the wave frequency $\hat{\omega}$ and excitation force $\hat{F}_{e x}$ are good). In fact:

- Velocity and excitation force are always in phase, independent of the model.

- The amplitude of the velocity is optimal in the constrained region, i.e. the second condition in (13), independent of the model.

- In the unconstrained region, i.e. the first condition in (13), the amplitude of the velocity is suboptimal and depends on the approximation $2 B(\omega)+2 K_{v}^{(0)}$.

As already studied in [8], particularly in large waves (more of interest for energy extraction), WECs will mostly work in a constrained regime, so that the reference-generation strategy in (13) will only depend on the model for relatively small waves, where the linear model is more accurate. In this sense, we consider the proposed high-level controller to be weakly dependent on the model. Moreover, knowledge of the response of the non-linear system at excitations of different magnitude could be incorporated in (13) to improve the accuracy of the amplitude relation, by adapting $K_{v}^{(0)}$ as a function of both $\hat{\omega}$ and $\hat{F}_{e x}$, for example by means of a 


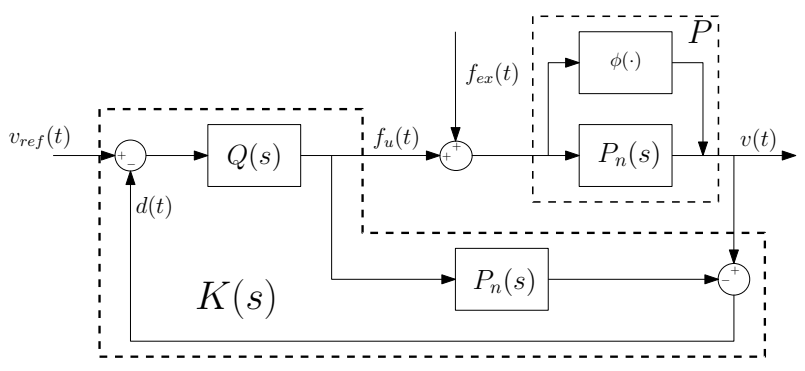

Fig. 4. Internal Model Control (IMC) structure [19].

look-up-table and experimental data of the type shown in Fig. 2.

Note that alternative choices based, for example, on modifications of the classical MPC [9]-[12] could be considered in the production of a velocity set-point, but these are not considered, here, since the main focus of this paper is on the servo-controller loop.

\section{B. Low-level feedback control}

The low-level servo controller is designed based on the principle of internal model control (IMC) [19], shown in Fig. 4. The feedback signal, $d(t)$, can be interpreted as the difference between the output of the system, $P$, and the expected output based on the nominal model, $P_{n}$. IMC exploits the idea that if a process and all its inputs are known perfectly, there is no need for feedback control or, in other words that, for stable processes, feedback control is only needed because of uncertainty [19]. Note that IMC was already proposed in the context of wave energy, [20], but the robust design was not explicitly addressed.

It can be easily shown [19] that the conceptual scheme in Fig. 4 is equivalent to the classical feedback loop of Fig. 3, when:

$$
K(s)=\frac{Q(s)}{1-P_{n}(s) Q(s)} .
$$

As is typical in control systems design, in (14) and in the following, $s \in \mathcal{C}$ denotes the complex frequency in the Laplace domain, on which transfer functions of finite-order linear systems are defined. The nominal model $P_{n}(s)$ is a finite order approximation of $P_{n}(\omega)$, defined in (8), such that $P_{n}(s=\jmath \omega) \approx P_{n}(\omega)$. The approximation is based on a finite-order approximation of the system radiation defined in [21] and more details can also be found in [8].

While $K(s)$ is the actual feedback controller to be implemented in practice, the filter $Q(s)$ is the only design parameter of the IMC controller. Due to the special structure of IMC, $Q(s)$ can be quite intuitively chosen, based on performance and robustness specifications, as will emerge in the following.

The relation between the output, $v(t)$, the control input, $v_{r e f}(t)$, and the external excitation (disturbance), $f_{e x}(t)$, of the system in Fig. 4 is:

$$
\begin{array}{r}
v=\frac{P Q}{1+Q\left(P-P_{n}\right)} v_{r e f}+\frac{1-P_{n} Q}{1+Q\left(P-P_{n}\right)} P f_{e x} \\
=T v_{r e f}+S P f_{e x}
\end{array}
$$

where the dependence on the complex variable, $s$, has been dropped for brevity. The sensitivity function, $S(s)$, and the complementary sensitivity function, $T(s)$, are defined [19] as:

$$
\begin{aligned}
& S \triangleq \frac{1-P_{n} Q}{1+Q\left(P-P_{n}\right)}=\frac{v}{P f_{e x}} \\
& T \triangleq \frac{P Q}{1+Q\left(P-P_{n}\right)}=\frac{v}{v_{\text {ref }}} .
\end{aligned}
$$

The sensitivity function expresses the response of the feedback system to the external disturbance. The complementary sensitivity indicates the tracking ability of the closed-loop system, as well as the sensitivity to measurement disturbances [19]. When the plant coincides with the nominal model, $P_{n}(s)=P(s)$, the ideal choice for $Q(s)$ is:

$$
\widetilde{Q}(s)=P_{n}^{-1}(s),
$$

such that perfect control is achieved: $S=0$ (perfect disturbance rejection) and $T=1$ (perfect reference tracking). However, the resulting $K=\widetilde{Q} /\left(1-P_{n} \widetilde{Q}\right)$ is not physically realisable, since $P_{n}(s)$ can be shown to be strictly proper, with relative degree 1 , and non-minimum phase, with a zero at $s=0$ [8], [15].

Therefore, the filter $Q(s)$ is augmented as:

$$
\widetilde{Q}(s)=F(s) P_{n}^{-1}(s),
$$

where $F(s)$ should be proper with relative degree of at least 1 and a zero at $s=0$, in order to remove the unstable pole at $s=0$ appearing from the inversion of $P_{n}(s)$. As already proposed in [8], and similarly in [20], $F(s)$ is designed as follows:

$$
F(s)=\frac{s}{(s+0.2)} \cdot \frac{5}{s+5},
$$

i.e., a band-pass filter which approximates perfect control in the frequency range $[0.3,2] \mathrm{rad} / \mathrm{s}$ (wave period of $[21,6.28]$ s). Note that this choice of $F(s)$ is somewhat arbitrary.

The controller thus-far obtained, namely $\widetilde{K}(s)$, does not ensure closed-loop stability because the nominal model, $P_{n}(s)$, is only a linear approximation of the actual behavior of the WEC. In the following, we investigate an approach to adjust $\widetilde{K}(s)$ such that the final controller, $K(s)$, yields a robustly-stable feedback loop.

1) Robustness based on passivity: An important result from input-output stability theory is the passivity theorem, confirming that the feedback interconnection of a passive system and a strictly passive one is always input-output stable [22], [23].

Definition 1: A (possibly non-linear) system $y(t)=$ $f(\dot{y}, u, t)$ is passive if $\int_{0}^{T} y(t) u(t) d t \geq 0 \forall T \geq 0$ and for any $u(t)$.

Definition 2: A function $G(s)$ of a complex variable $s$ is positive real if $G(s)$ is analytic for $\Re\{s\}>0, G(s)$ is real for real $s$ and $\Re\{G(s)\} \geq 0 \forall \Re\{s\}>0$. We say that $G(s)$ is strictly positive real (SPR) if $\Re\{G(s)\}>0 \forall \Re\{s\}>0$ A linear time-invariant system is (strictly) passive if its transfer function is (strictly) positive real [23]. 


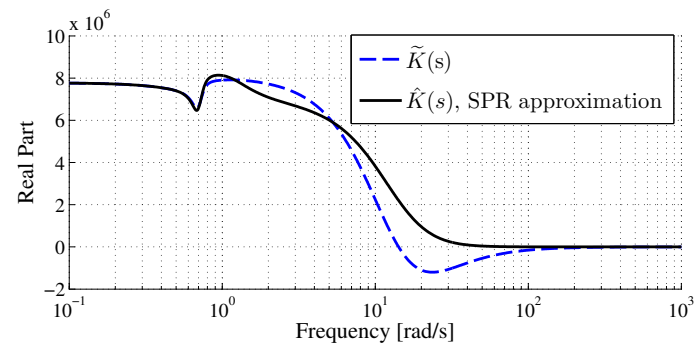

Fig. 5. SPR approximation of the nominal controller, $\widetilde{K}(s)$, based on (21).

Based on Definition 1, a passive system always dissipates energy (energy always positive). It can be immediately verified that the non-linear model of the WEC, given in (7), is passive. The linear part of the system (radiation and buoyancy effects) is positive real [3], [21] and therefore passive, while the non-linear viscous force in (6) is passive, since $f_{v}(t) \cdot v(t)>0$ always. In addition, other non-linear effects that may arise in the generic WEC model, given in (1), are all of a dissipative nature.

Based on the passivity theorem, it is therefore sufficient to design a SPR $K(s)$ in order to ensure closed-loop stability. While it is difficult to approach the design of a controller with the SPR constraint, it is indeed possible to obtain a SPR approximation of an initial controller, $\widetilde{K}(s)$. In particular, the nominal controller obtained in (19) and (14) is approximated based on the procedure proposed in [23]:

$$
\begin{aligned}
\hat{K}(s)= & \operatorname{argmin} \frac{1}{2 \pi} \int_{-\infty}^{+\infty}|\widetilde{K}(\jmath \omega)-K(\jmath \omega)|^{2} d \omega \\
& \text { subject to: } \quad K(s) \text { is } S P R .
\end{aligned}
$$

The SPR constraint is formally expressed in terms of the coefficients of the denominator of the transfer function $K(s)$ and the problem in (21) is solved with non-linear convex programming [23]. Figure 5 compares the real parts of $\widetilde{K}(s)$ (negative at times) and the resulting $K(s)$ (always positive).

Note that the design does not need any knowledge of the non-linearity of the system. In addition, one can increase the gain of $K(s)$ while not worrying about the closed-loop stability ( $K$ is still SPR) and independently improve the reference-tracking and excitation-force-rejection properties of the servo controller. We apply an additional multiplicative gain of 20 to the final $K(s)$, based on intuitive design (the gain is increased until the velocity tracking is acceptable). The resulting controller is referred to as $K_{S P R}(s)$ :

$$
K_{S P R}(s)=20 \hat{K}(s) \text {. }
$$

\section{Results}

\section{A. Wave data}

Simulation results are produced under several wave conditions. Random waves are generated from single-peaked, three-parameters Ochi spectral distributions [24]:

$$
S_{\eta \eta}(\omega)=\frac{\left(\frac{4 \lambda+1}{4} \omega_{0}^{4}\right)}{\Gamma(\lambda)} \cdot \frac{H_{s}^{2}}{\omega^{4 \lambda+1}} e^{-(4 \lambda+0.25)\left(\omega_{0} / \omega\right)^{4}},
$$

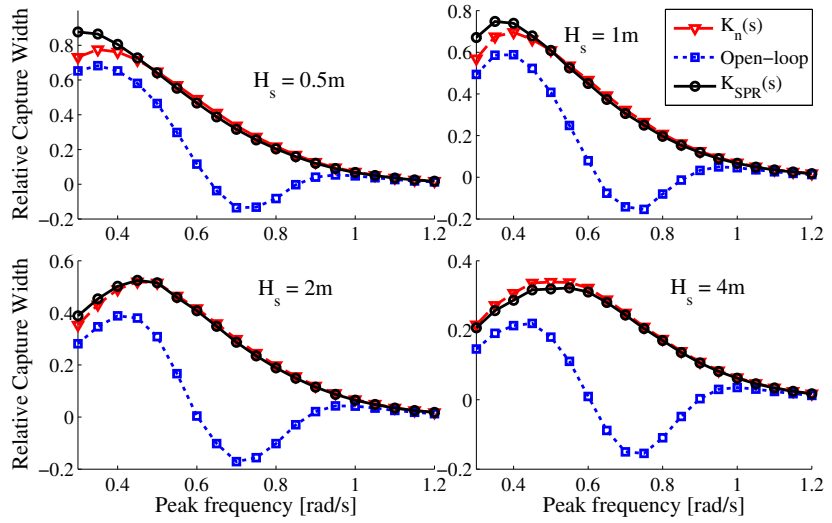

Fig. 6. Performance of the proposed robust controller against the open-loop controller based on the nominal model.

where $\omega_{0}$ is the peak frequency, $H_{s}$ is the significant wave height and $\lambda$ is the sharpness.

The peak frequency is varied from 0.3 to $1.2 \mathrm{rad} / \mathrm{s}$, with step 0.05 , the significant wave heights are $0.5,1,2$ and $4 \mathrm{~m}$, and $\lambda=5$ (not important for the purposes of this study; the reader may refer to [8] to verify how the bandwidth affects the high-level control strategy). Random-wave time series of $7200 \mathrm{~s}$, sampled at $2.56 \mathrm{~Hz}$, are generated for each simulation, from $S_{\eta \eta}(\omega)$, based on the method proposed in [25] and also described in [8].

\section{B. Results}

The performance of the hierarchical control system of Fig. 3 is evaluated across the range of waves described in section IV-A, over which the linear model of WEC has a quite limited accuracy, as highlighted in Fig. 2. By way of comparison, for the servo-controller component, we consider the nominal controller designed in section III-B, denoted as $K_{n}(s)$, although this should not be implemented in practice, since it could cause instability.

A first general measure of the robustness, shown in Fig. 6 , is the overall energy extraction, in terms of relative capture width (RCW) [8], obtained by the controllers over the whole range of wave conditions. Clearly, the openloop nominal controller produces unwanted results (negative energy extraction) over a wide range of frequencies around resonance $(0.7 \mathrm{rad} / \mathrm{s})$, where the largest model uncertainty is. Both of the proposed servo-controllers, on the other hand, are clearly robust in all sea conditions, producing the expected RCW. $K_{S P R}(s)$ is seen to be superior, particularly for lowfrequency waves, and this is due to the superior velocitytracking performance, highlighted in section III-B. Figure 7 shows the lower loop velocity tracking performance of $K_{S G}(s)$. Clearly, there is minimal velocity tracking error.

The importance of accurately following the desired reference velocity is not only evident in the energy-capture performance of the WEC, but also in the ability to satisfy the desired motion constraints. The proposed strategy for the generation of the velocity setpoint, given in (13), also accounts for the motion constraint $X_{\text {lim }}= \pm 2 \mathrm{~m}$. Figure 8 


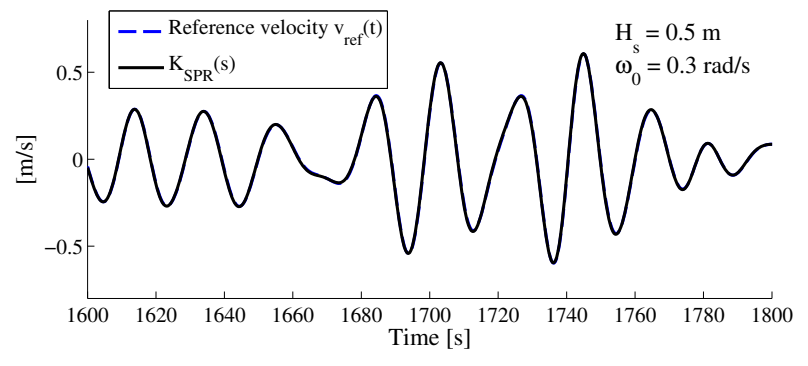

Fig. 7. Example of velocity-tracking performance of the proposed controllers. Note that $v_{r e f}(\mathrm{t})$ is indistinguishable from the velocity produced by $K_{S P R}(s)$.

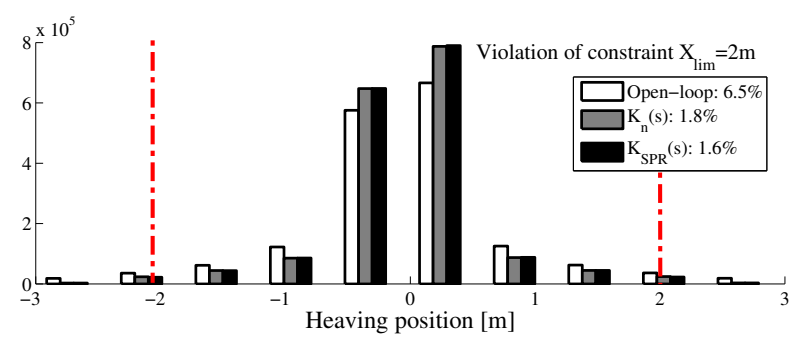

Fig. 8. Distribution of the WEC heaving position with the different proposed controllers.

shows the distribution of the heaving position over the whole range of proposed simulations. The given constraint is exceeded about $6.5 \%$ of the times with the open-loop nominal controller, while less than $2 \%$ of the times with the proposed robust controllers. Given the very accurate reference-tracking performance of the controller $K_{S P R}(s)$, such constraint violations are mostly due to the monocromatic time-varying approximation of key system variables and instantaneous frequency/amplitude estimation of the high-level controller, already discussed in [8].

\section{CONCLUSION}

The adopted procedure seeks a sensible trade-off between performance and simplicity, resulting in a fixed parameter controller with no requirement for wave forecasting, while directly addressing linear modelling errors due to non-linear viscosity effects. The design procedure is both intuitive and straightforward; yet, physical system constraints can be directly addressed. While the performance of the resulting controller is sub-optimal, with some conservatism due to the robust formulation, we feel that the performance penalty over a nonlinear controller which deals explicitly with system nonlinearities is more than compensated by the linear controller simplicity.

\section{REFERENCES}

[1] M. T. Pontes and A. Brito-Melo, "International Energy Agency Cooperate R\&D on Ocean Energy," in Proceedings of the 5th European Wave Energy Conference, Cork, Ireland, 2003, pp. 10-13.
[2] J. Cruz, Ocean wave energy. Current status and future perspectives. Springer-Verlag, 2008.

[3] J. Falnes, Ocean waves and oscillating systems. Cambridge University Press, 2002.

[4] J.-C. Gilloteaux, "Mouvements de grande amplitude d'un corps flottant en fluide parfait. Application à la récupération de l'énergie des vagues," Ph.D. dissertation, Ecole Centrale de Nantes, France, 2007.

[5] A. Merigaud, J.-C. Gilloteaux, and J. V. Ringwood, "A Nonlinear Extension for Linear Boundary Element Methods in Wave Energy Device Modelling," in Proceedings of the 31st International Conference on Ocean, Offshore and Arctic Engineering (OMAE), Rio de Janeiro, Brazil, 2012.

[6] M. A. Bhinder, A. Babarit, L. Gentaz, and P. Ferrant, "Assessment of Viscous Damping via 3D-CFD Modelling of a Floating Wave Energy Device," in Proceedings of the 9th European Wave and Tidal Energy Conference (EWTEC), Southampton, UK, 2011.

[7] Z. Yuan and Z. Huang, "An experimental study of inertia and drag coefficients for a truncated circular cylinder in regular waves," Journal of Hydrodynamics, vol. 22, no. 5, pp. 318-323, 2010.

[8] F. Fusco and J. V. Ringwood, "A Simple and Effective Real-time Controller for Wave Energy Converters," IEEE Transactions on Sustainable Energy, vol. 4, no. 1, pp. 21-30, 2013.

[9] J. Hals, J. Falnes, and T. Moan, "Constrained Optimal Control of a Heaving Buoy Wave-Energy Converter," Journal of Offshore Mechanics and Arctic Engineering, vol. 133, no. 1, p. 011401, 2011.

[10] G. Bacelli, J. V. Ringwood, and J.-C. Gilloteaux, "A control system for a self-reacting point absorber wave energy converter subject to constraints," in Proceedings of the 18th IFAC World Congress, Milano, Italy, 2011, pp. 11387-11392.

[11] J. A. M. C. G. Lightbody, "Maximisation of Energy Capture by a Wave-Energy Point Absorber using Model Predictive Control," in Proceedings of the 18th IFAC World Congress, no. 2002, Milano, Italy, 2011, pp. 3714-3721.

[12] M. Richter, M. E. Magaña, S. Member, O. Sawodny, and T. K. A. Brekken, "Nonlinear Model Predictive Control of a Point Absorber Wave Energy Converter," IEEE Transactions on Sustainable Energy, vol. 4, no. 1, pp. 118-126, 2013.

[13] J.-C. Gilloteaux, G. Bacelli, and J. V. Ringwood, "A non-linear potential model to predict large-amplitudes-motions: application to a multi-body wave energy converter," in Proceedings of the 10th World Renewable Energy Congress (WREC X), Glasgow, Scotland, 2010.

[14] J. R. Morison, M. P. O'Brien, J. W. Johnson, and S. A. Schaaf, "The Force Exerted by Surface Waves on Piles," AIME Petroleum Transactions, vol. 189, pp. 149-154, 1950.

[15] F. Fusco and J. V. Ringwood, "A Study on the Prediction Requirements in Real-Time Control of Wave Energy Converters," IEEE Transactions on Sustainable Energy, vol. 3, no. 1, pp. 176-184, 2012.

[16] Wamit Inc., "WAMIT Vers. 6.4," 2008.

[17] D. Atherthon, Nonlinear control engineering. Van Nostrand Reinhold, 1982.

[18] F. Fusco and J. V. Ringwood, "Short-Term Wave Forecasting for RealTime Control of Wave Energy Converters," IEEE Transactions on Sustainable Energy, vol. 1, no. 2, pp. 99-106, Jul. 2010.

[19] M. Morari and E. Zafiriou, Robust Process Control. Prentice-Hall, Inc., 1989.

[20] J. Sá da Costa, P. Beirão, and D. Valério, "Internal Model Control Applied to the Archimedes Wave Swing," in 16th Int. Conf. on Control Systems and Comp. Sci. (CSCS), Bucharest, Romania, 2007.

[21] T. Perez and T. I. Fossen, "A Matlab Toolbox for Parametric Identification of Radiation-Force Models of Ships and Offshore Structures," Model Identification and Control, vol. 30, no. 1, pp. 1-15, 2009.

[22] C. A. Desoer and M. Vidyasagar, Feedback systems: input-outpu properties. Academic Press, New York, 1975.

[23] C. Damaren, H. Marquez, and A. Buckley, "Optimal strictly positive real approximations for stable transfer functions," IEE Proc. - Control Theory and Apps., vol. 143, no. 6, pp. 537-542, Nov. 1996.

[24] M. K. Ochi and E. N. Hubble, "Six-parameter Wave Spectra," Proceedings of the 15th International Conference on Coastal Engineering, 1976.

[25] J. Figwer, "A new method of random time-series simulation," Simulation Practice and Theory, vol. 5, pp. 217-234, 1997. 